\title{
First report of grapevine enamovirus 1 in grapevine in China
}

\author{
Fang Ren ${ }^{1} \cdot$ Zun Ping Zhang ${ }^{1} \cdot$ Xu Dong Fan ${ }^{1} \cdot$ Guo Jun $\mathrm{Hu}^{1} \cdot$ Ya Feng Dong ${ }^{1}$ (])
}

Received: 28 July 2020 / Accepted: 5 October 2020 / Published online: 15 October 2020

(C) Società Italiana di Patologia Vegetale (S.I.Pa.V.) 2020

Keywords Grapevine · Grapevine enamovirus-1 · Complete genome

Grapevine enamovirus 1(GEV1) is a member in the genus Enamovirus in the family Luteoviridae. It was described for the first time in four grapevine (Vitis vinifera L.) cultivars in Brazil (Silva et al. 2017). In October 2019, a 'Cabernet Gernischt' grapevine collected from Shandong province in China displaying chlorotic mottling symptom was analyzed by high-throughput sequencing with Illumina HiSeq X-ten platform. A total of 31,686,103 cleaned reads were obtained, and 10,435 contigs were generated, among which a contig $(6,317 \mathrm{bp})$ showed $89.55 \% \mathrm{nt}$ identity with GEV1 isolate CS-BR (KX645875) via Blastx comparisons. The presence of GEV1 was further confirmed by RT-PCR and sequencing using four reported primer pairs Set1F/1R, Set2F/2R, Set3F/ 3R and Set6F/6R (Silva et al. 2017). The complete genome of GEV1 SD-CG isolate was obtained by three primer pairs, GEV-1F/1R (5'-TTGCCTCCTTATGCTCACTC-3'/5'AATAGGTCAACATTCG CTCC-3'), GEV-2F/2R (5'GTGGGCAGGCAGATGTAA-3'/5'-AACCAGTG TTTGGCGAAG TA-3'), and GEV-3F/3R (5'-TTCCCTTG GGAGACTCGGTTCTGT-3'/5'-AAACATGACTACCC GTCTCATAGC-3') and rapid amplification of cDNA ends using SMARTer RACE 5'/3' Kit (Takara, China). The complete genome was deposited in GenBank under accession No. MT536978. It comprises 6,317 nt, contains five open reading frames and shows $89.6 \%$ and $89.4 \%$ identities with GEV1 isolates CS-BR and SE-BR (KY820716), respectively.
It is 93-nt and 144-nt longer than isolates CS-BR and SE-BR, respectively, and has a 9-nt deletion in the $3^{\prime}$ untranslated region and a 3-nt insertion in the overlapping area of ORF1 and ORF2. A neighbor joining tree for member of the family Luteoviridae based on the complete genome sequence showed that GEV1 isolate SD-CG clustered together with isolates CS$\mathrm{BR}$ and SE-BR but grouped in a distinct clade, indicating that it is likely to be a new variant of GEV1. GEV1 was detected by RT-PCR in $0.5 \%$ ( 2 of 403) grapevine samples of 68 cultivars from 20 provinces in China, indicating it might not be common. To our knowledge, this is the first report of GEV1 infecting grapevine in China.

Funding This work was supported by the China Agriculture Research System (CARS-29-bc-1), National key R\&D Program of China (2019YFD1001800), and Natural Science Foundation of Liaoning Province of China (2020-MS-035).

\section{References}

Silva JMF, Al Rwahnih M, Blawid R, Nagata T, Fajardo TVM (2017) Discovery and molecular characterization of a novel enamovirus, Grapevine enamovirus-1. Virus Genes 53:667-671

Publisher's note Springer Nature remains neutral with regard to jurisdictional claims in published maps and institutional affiliations.
Ya Feng Dong

yfdong@163.com

1 National Center for Eliminating Viruses from Deciduous Fruit Trees, Research Institute of Pomology, Chinese Academy of Agriculture Sciences, Liaoning 125100 Xingcheng, China 